\title{
Structure of Reactive Intermediates of Organocatalysis
}
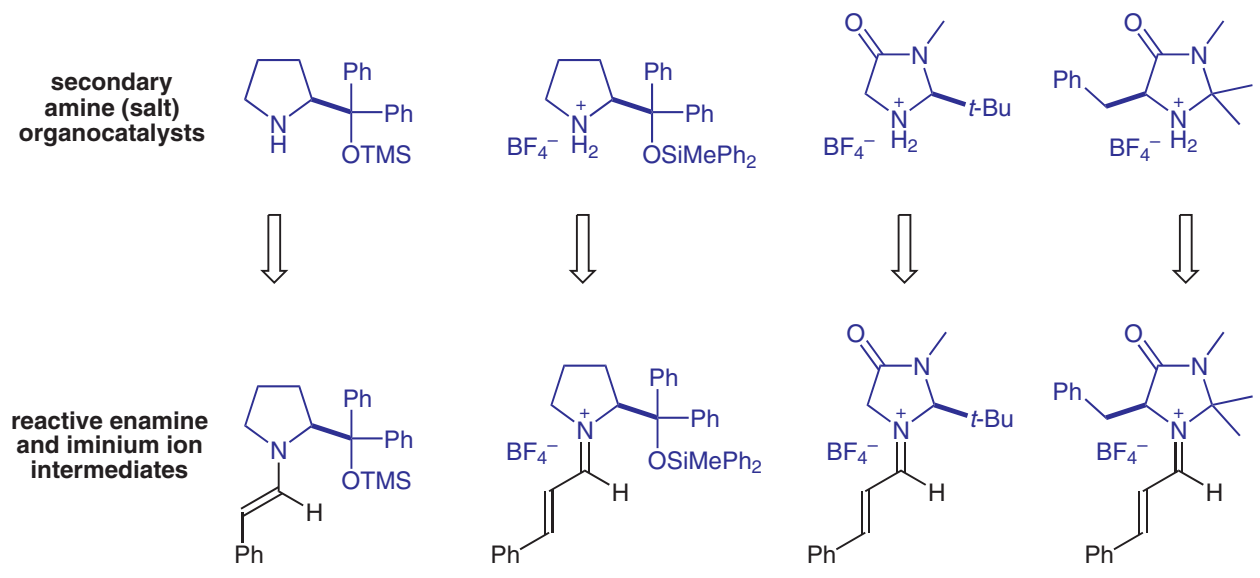

1

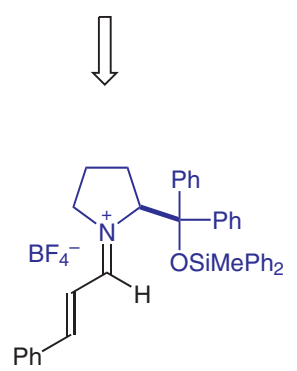

2<smiles>C=CC1N(C)C(=O)CN1C(Br)=CCc1ccccc1</smiles>

3<smiles>C=CC=CC(=O)N1C(=O)C(Cc2ccccc2)N(C)C1(C)C</smiles>

4

NMR analysis
Key words

reactive

intermediates

enamines

iminium ions

structure

elucidation
Significance: The present work of the Seebach group addresses the isolation and characterization of reactive intermediates in organocatalysis. In this regard, enamine $\mathbf{1}$ and iminium tetrafluoroborates 2-4 were prepared through conventional methods from 2-phenyl acetaldehyde or cinnamaldehyde, respectively, and the corresponding secondary amine (salt) catalyst. Single crystals of enamine $\mathbf{1}$ and iminium ions $\mathbf{2}$ and $\mathbf{3}$ were subjected to Röntgen structure analysis. The structure of iminium ion 4 was elucidated by 2D NMR spectroscopy. Moreover, the Röntgen and NMR structures were extensively discussed in connection with other, structurally related compounds.
Comment: The experimentally determined NMR and Röntgen structures of 1-4 were also compared with computed structures, and the similarity is striking (for DFT calculations of iminium ions similar to $\mathbf{3}$ and $\mathbf{4}$ derived from crotonaldehyde, see: R. Gordillo, H. Carter, K. N. Houk Adv. Synth. Catal. 2004, 346, 1175). In fact, Seebach et al. confirmed structural effects predicted by theory now experimentally. These results suggest that in future synthetic organic chemists will more and more rely on computational methods for finding the best (organo)catalyst instead of carrying out tedious experimental optimizations. Similar studies might also be desirable for other classes of organocatalysts such as primary amine (salt) catalysts. 